Transient photocurrent response of three-color detectors based on amorphous silicon

B. Stannowski, H. Stiebig, D. Knipp, and H. Wagner

Citation: Journal of Applied Physics 85, 3904 (1999);

View online: https://doi.org/10.1063/1.369762

View Table of Contents: http://aip.scitation.org/toc/jap/85/7

Published by the American Institute of Physics

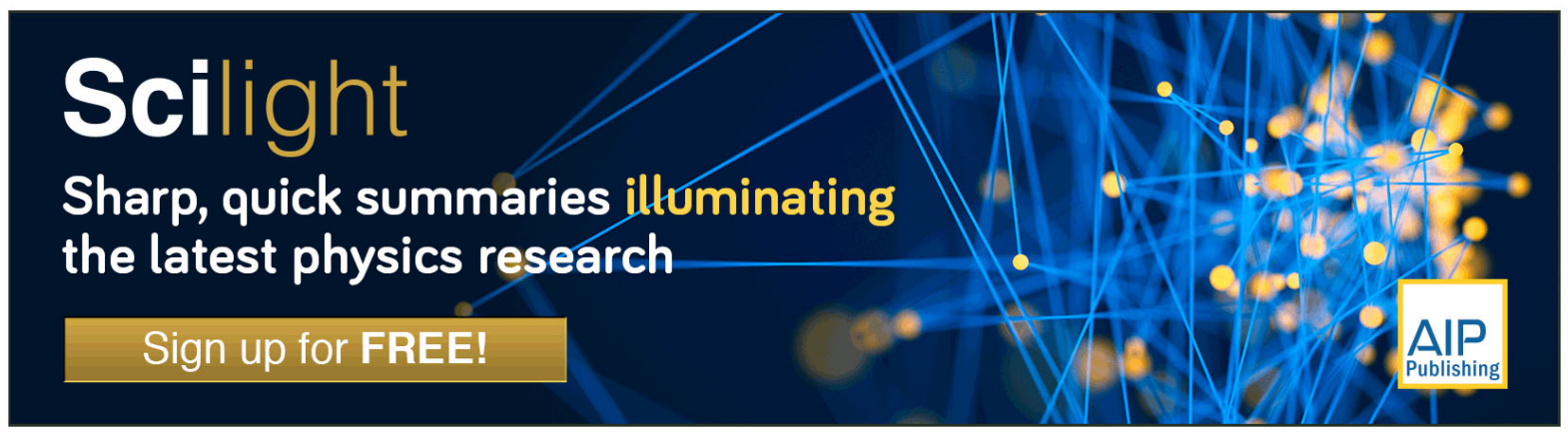




\title{
Transient photocurrent response of three-color detectors based on amorphous silicon
}

\author{
B. Stannowski, ${ }^{\text {a) }}$ H. Stiebig, D. Knipp, and H. Wagner \\ Institute of Thin-Film and Ion Technology, Research Centre Jülich, 52425 Jülich, Germany
}

(Received 1 October 1998; accepted for publication 4 January 1999)

\begin{abstract}
Color detectors based on multilayers of amorphous-silicon alloys facilitate the detection of the three fundamental components of visible light in one single pixel of a sensor array. In order to achieve sensitivity for the blue, green, and red components of light, three different bias voltages are applied to the device. By switching them sequentially the detector is read out. $n-i-p-i-i-n$ structures with a controlled band gap and mobility-lifetime product exhibit excellent stationary properties, namely: good color separation and have dynamic behaviors above $95 \mathrm{~dB}$. Besides the stationary behavior the transient response of a color detector is a further optimization criterion. The experimentally found transient photocurrent response after switching on monochromatic light at different applied bias voltages showed reasonable delay times in the range of tens of milliseconds before reaching steady state. Numerical simulations have been carried out which reproduce this characteristic behavior and facilitate a study of time dependent processes within the device, such as charge transport and storage in localized states. The delay times can be explained by the recharging of electrical defect states in the amorphous material. Consequently, the electrical potential within the device changes, which remarkably affects the carrier transport. Based on these results optimization criteria for the transient behavior of the color detectors are discussed. (c) 1999 American Institute of Physics. [S0021-8979(99)03507-0]
\end{abstract}

\section{INTRODUCTION}

Hydrogenated amorphous silicon $(a-\mathrm{Si}: \mathrm{H})$ based thin film detectors can be applied for color recognition, since $a-\mathrm{Si}: \mathrm{H}$ and related materials exhibit a high photosensitivity and a strong wavelength dependent absorption coefficient between the near ultraviolet (UV-A) and the near infrared (IR) part of the spectrum. Due to the wavelength dependent penetration depth of incident light, the spatial resolution of photogenerated carriers offers good feasibilities for color separation and, thus, detection of the fundamental components of visible light. Such $a$-Si:H based devices can be produced by plasma enhanced chemical vapor deposition (PECVD) at low temperatures between 200 and $300^{\circ} \mathrm{C}$ on various substrates like glass, steel, or crystalline silicon. The deposition technology allows an easy modification of the material properties by adding methane $\left(\mathrm{CH}_{4}\right)$ or germane $\left(\mathrm{GeH}_{4}\right)$ to the process gas silane $\left(\mathrm{SiH}_{4}\right)$, thereby depositing the alloys $a-\mathrm{SiC}: \mathrm{H}$ or $a$-SiGe:H. By this technology various vertical integrated color sensitive devices like $n-i-p-i-n,{ }^{1,2} p-i-n-i-p,{ }^{3} p-i-n$ with modified $i$-layer profiles, ${ }^{4,5}$ and even more complex device structures ${ }^{6,7}$ have been produced. The preferential carrier collection region of these two-terminal devices shifts upon changing an applied voltage, which leads to a color sensitivity. The spectral response of the devices varies from red to green and blue (RGB signal). The combination of a thin-film color detector with amorphous-, polycrystalline-, or crystalline-silicon read-out electronics can be used to produce color sensitive sensor arrays, which are already realized for black and white sensors. ${ }^{8-11}$ Since the RGB signal in

\footnotetext{
${ }^{a)}$ Electronic mail: b.stannowski@phys.uu.nl
}

those devices is detected at the same spatial detector position, a high area fill factor can be achieved and the colormoiré effect can be prevented. Therefore, each detector is read out sequentially, which requires a fast transient behavior. In order to develop optimization criteria to achieve adequate color detection with high frame rates, more insight into the transport and recombination behavior of carriers in a color-sensor structure after light exposure is necessary. Therefore, the transient photocurrent response of color detectors has been a matter of numerous investigations. Since up to now only the stationary behavior of $n-i-p-i-n$ and $p-i-n-i-p$ structures has been investigated by numerical modeling, ${ }^{2,7,12}$ the simulation of the transient response can be a useful tool to explain the limiting processes which avoid high-speed color detection.

In this study we focus on the transient behavior of a high-quality $n-i-p-i-i-n$ three-color detector based on $a-\mathrm{Si}: \mathrm{H}$ alloys by comparing experimental and numerically simulated data. Due to an improved device design our detector exhibits good color separation, bias-light independent spectral response curves, and a linear relation between photon flux and photocurrent. It exhibits a high dynamic range exceeding $95 \mathrm{~dB},{ }^{4}$ which is defined as the ratio between the current under $10001 \times$ white-light illumination and the dark current: $20 \times \log \left(I_{\text {light }} / I_{\text {dark }}\right)$. However, the sensor performance is limited by the response time, especially at low levels of illumination intensity. ${ }^{13-15}$ While extensive studies of the transient response after bias-voltage switching were carried out and described elsewhere, ${ }^{16}$ we have investigated the transient photocurrent response after switching on a constant illumination. 


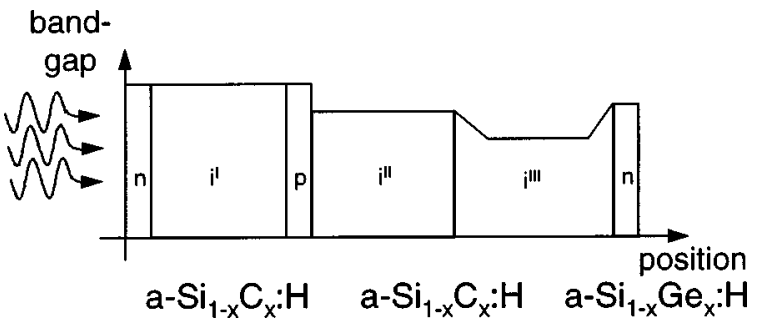

FIG. 1. Band gap structure of a $n-i-p-i-i-n$ three-color detector (schematically).

\section{EXPERIMENT}

The color sensitive diodes discussed here have been deposited in a multichamber PECVD system on glass substrates coated with smooth transparent conductive oxide (TCO). The deposition was conducted at a substrate temperature of $200{ }^{\circ} \mathrm{C}$, a pressure of 500 mTorr, and a rf power density of $35 \mathrm{~mW} / \mathrm{cm}^{2}$. As a rear contact of the multilayer structure, thermally evaporated aluminum was used. In order to achieve $10 \mathrm{~mm}^{2}$ test-pixel structures, the samples were patterned using photolithography and reactive-ion etching. Figure 1 sketches the layer sequence of the $n-i-p-i-i-n$ structure. It consists of six layers with thicknesses of 13,125 , $20,200,100$, and $20 \mathrm{~nm}$. The optical band gaps of the different intrinsic absorption layers decrease from 2 to 1.9 to $1.55 \mathrm{eV}$. This band gap profiling is achieved by alloying carbon or germanium to the $a-\mathrm{Si}: \mathrm{H}$ layers using process-gas mixtures of silane, methane, and germane. The different materials also result in a variation of the mobility-lifetime produce $\mu \tau$. With increasing Ge content an increase of the defect density and a reduction of the $\mu \tau$ produce is observed. ${ }^{17,18}$ The doped layers were deposited by adding phospine for $n$-type material and trimethylboron for $p$-type material to the process gas.

The transient response measurements were performed by switching red or blue light emitting diodes (LEDs), respectively, which are arranged in an array. These light sources facilitated rise times of less than $5 \mu$ s and a photon flux of $10^{15} \mathrm{~cm}^{-2} \mathrm{~s}^{-1}$. In combination with interference filters wavelength spectra of the incident light with maxima at $650 \mathrm{~nm}$ $(450 \mathrm{~nm})$ and widths of $50 \mathrm{~nm}$ were achieved. The response time of the entire setup was less than $10 \mu$ s. All measurements were performed at room temperature.

\section{NUMERICAL MODEL}

In the numerical simulations the complete set of time dependent semiconductor equations for electrons and holes, together with the rate equations which describe the interchange of charge carriers between extended and localized states in the band gap, are solved numerically. This is performed by an implicit method within a finite-difference scheme. The transport is assumed to take place via the extended states only. The high defect density of states positioned at mid-band gap (dangling bonds) typical for amorphous semiconductors, requires an accurate model for the density of states to describe carrier trapping and recombination as well as space charge effects. Therefore, a defect den-

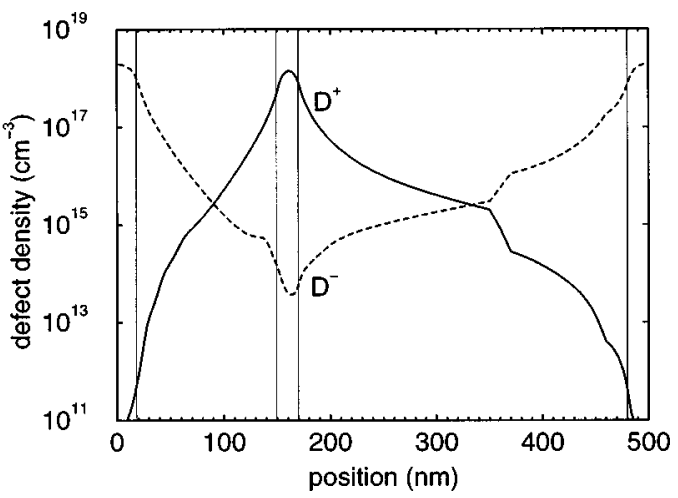

FIG. 2. Simulation results of charged defect states in a $n-i-p-i-i-n$ color detector in equilibrium.

sity resembling the defect-pool model was implemented. In order to describe a nonequilibrium situation we divide the density of dangling-bond states into three Gaussian defect peaks: $D^{n}, D^{i}$, and $D^{p}$. The energy position of $D^{n}$ is located in the lower half, $D^{i}$ lies close to mid-band gap, and $D^{p}$ is positioned in the upper half of the band gap. According to the amphoteric character of dangling-bond states, each state can be occupied by two, one, or no electrons resulting in a negative, neutral, or positive charge state. Thus, in equilibrium these three defect bands $\left(D^{n}, D^{i}, D^{p}\right)$ are charged negatively, neutrally, and positively, respectively. Taking into account the positive correlation energy $U$ between two electrons occupying the same defect state, negatively charged states $D^{-}$are assumed to be separated from positive and neutral states $D^{+} D^{0}$ by $U=0.2 \mathrm{eV}$. The separation energy between the positively charged defect states of the $D^{p}$ peak and the negatively charged defect states of the $D^{n}$ peak have been chosen to be independent from the optical gap used. In order to keep the calculation time reasonably short, each of the defect peaks $\left(D^{n}, D^{i}\right.$ and $\left.D^{p}\right)$ is represented by seven discrete defect levels. Values for the Fermi level dependent defect density in $a-\mathrm{Si}: \mathrm{H}$ and $a-\mathrm{SiGe}: \mathrm{H}$ capture cross sections and the free-carrier mobilities of electrons (10 $\left.\mathrm{cm}^{2} / \mathrm{V} \mathrm{s}\right)$ and holes $\left(2 \mathrm{~cm}^{2} / \mathrm{V} \mathrm{s}\right)$ were comparable to those used for simulation of constant photocurrent measurements (CPMs) and photothermal deflection spectroscopy (PDS) spectra. ${ }^{19,20}$ They were successfully applied for the simulation of solar cells based on $a$-Si:H (Ref. 21) and $a$-SiGe:H (Ref. 22) as well as for simulations of the transient photocurrent response of $a$-Si:H $p-i-n$ structures. ${ }^{23}$ The structure we used in our simulations is similar to the measured $n-i-p-i-i-n$ sequence with band gap profiling and two different $i$-layer materials in the bottom diode.

Figure 2 displays the self-consistently calculated defect distribution of the charged defect states in equilibrium $\left(D^{p}, D^{n}\right)$ caused by the spatially different position of the equilibrium Fermi level in the band gap. The defect density is dominated by negatively charged defect states in the $n$ layers and by positively charged states in the central $p$ layer. Since $a$-SiGe:H with a band gap of $1.5 \mathrm{eV}$ shows a higher defect density in comparison to high quality $a$-SiC:H, an enhanced negatively charged defect density is calculated in the rear part of the bottom diode. 


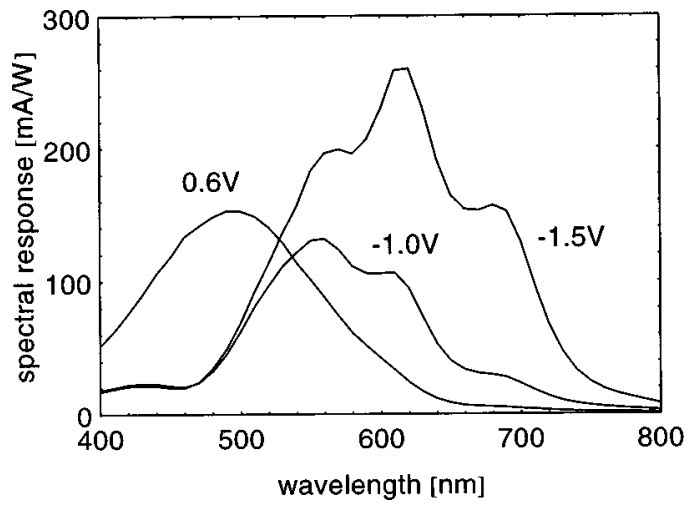

FIG. 3. Measured spectral response of a three-color detector with applied bias voltages of $+0.6,-1$, and $-1.5 \mathrm{~V}$.

\section{EXPERIMENTAL RESULTS}

The developed device design of the detector results in maxima of the spectral response at $620 \mathrm{~nm}$ (red), $550 \mathrm{~nm}$ (green), and $480 \mathrm{~nm}$ (blue) (Fig. 3). To read out the RGB signal the three voltages $-1.5,-1$, and $+0.6 \mathrm{~V}$ with respect to the grounded rear contact are switched sequentially. In the following, the $n-i-p$ sequence is denoted as the top diode, whereas the $p-i-i-n$ sequence in the rear part of the device is the bottom diode. The high band gap of the $i$-layer material in the top diode leads to a low absorption of light with longer wavelengths. Due to the high absorption coefficient (=short penetration depth) for light with shorter wavelengths, most carriers are generated in the front part of the structure, namely in the top diode under blue light illumination. By applying a positive voltage the top diode is reverse biased, collecting the photogenerated carriers and thus becomes blue sensitive. In this case a positive current is detected. In order to detect green and red light negative voltages are applied resulting in negative currents. To distinguish between the green and red light, which are both detected in the bottom diode, the absorption region of this diode is separated into two $i$ layers with different band gaps and material properties. As a result of the light absorption behavior the preferential carrier generation region shifts to the rear part with increasing wavelength. Application of low negative voltages results in a collection of electron-hole pairs generated in the front part of the bottom diode, whereas the generated carriers in the region with the low band gap in the rear part of the diode recombine. Therefore, green sensitivity of the detector is achieved. Only for higher negative voltages can nearly all photogenerated carriers in the bottom diode be extracted, resulting in red sensitivity.

Besides the above-described stationary behavior the transient response is an important criterion to characterize the detector performance. For amorphous silicon based devices after light or voltage switching delay times between a few hundred microseconds and some seconds depending on the photon flux, the dark time between the switching incidents as well as on the wavelength and the bias voltage have been observed. ${ }^{15}$ This behavior is also confirmed in Figs. 4 and 5, showing the transient photocurrent response of a $n-i-p-i-i-n$ color detector after switching on blue $(\lambda=450$

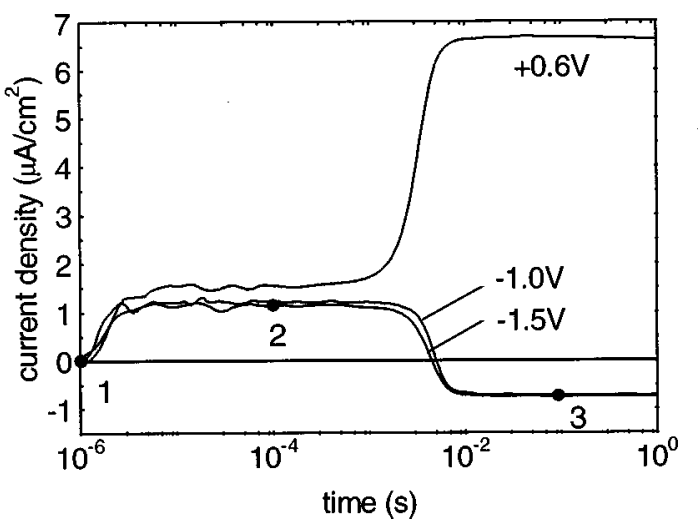

FIG. 4. Measured transient photocurrent response of a $n-i-p-i-i-n$ threecolor detector to switching on of illumination $(\lambda=450 \mathrm{~nm})$ with different applied bias voltages.

$\mathrm{nm})$ and red $(\lambda=650 \mathrm{~nm})$ illumination, respectively, with a preceding dark time of $10 \mathrm{~s}$. Three different bias voltages $V_{\text {bias }}=+0.6,-1$, and $-1.5 \mathrm{~V}$, typical for reading out the blue, green, and red signal, were applied. For all three voltages the curves exhibit considerable delay times up to several milliseconds before steady state is reached. These delay times are comparable with those found after voltage switching under constant illumination with similar light intensity. ${ }^{15,16}$

Figure 4 shows the photocurrent response after switching on blue illumination. In the dark (dot 1) the current is low due to the low rate of thermally excited carriers. After about $10^{-4} \mathrm{~s}(\operatorname{dot} 2)$ the photocurrent rises to a quasistationary value, which is nearly independent of the bias voltages. Finally, after a delay of some milliseconds, the current exhibits a stepwise rise to the steady state value for positive bias voltages, whereas a sign reversal of the signal is found for negative voltages of -1 and $-1.5 \mathrm{~V}$ (dot 3). The red response, shown in Fig. 5, exhibits features similar to the blue response, but with different voltage dependence. Again, a sign reversal is found for the current with the lowest stationary value, namely for a bias voltage of $+0.6 \mathrm{~V}$. Nevertheless, the current responses for the three bias voltages are less steep and in general more complex compared with the response after blue light switching.

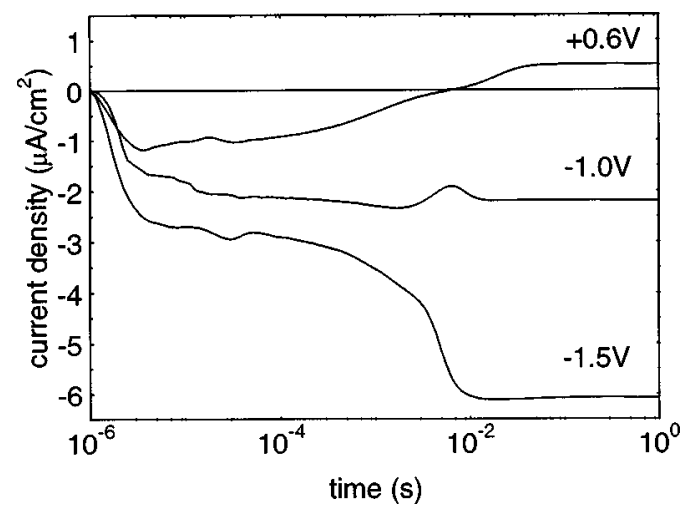

FIG. 5. Measured transient photocurrent response to switching on of illumination $(\lambda=650 \mathrm{~nm})$ with different bias voltages. 


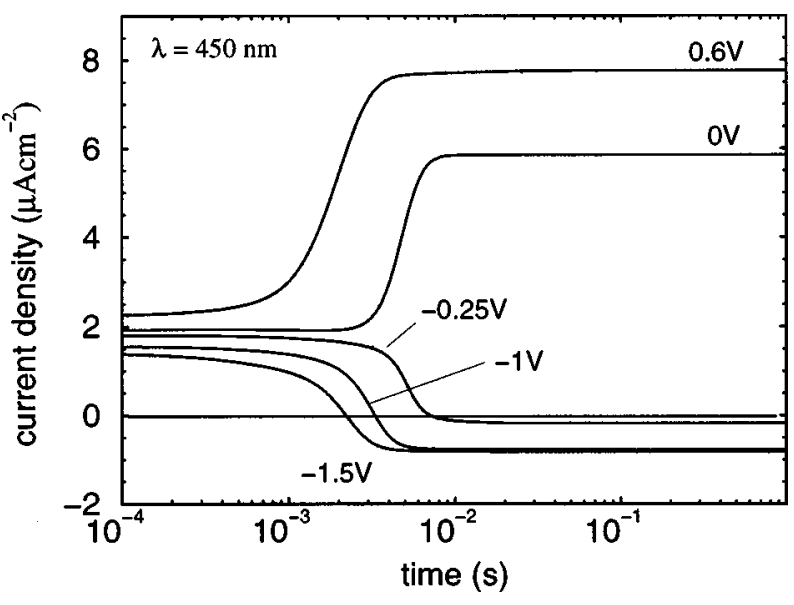

FIG. 6. Simulated transient photocurrent response to switching on of illumination $(\lambda=450 \mathrm{~nm})$ with different bias voltages.

Previous investigations have shown that the delay time of the transient photocurrent scales with illumination intensity, e.g., an increase of the photon flux by 1 order of magnitude leads to a current onset that is faster by nearly 1 order of magnitude. ${ }^{15}$ This behavior leads to the assumption that the charge, transferred through the device during light exposure, must be the same before steady state is reached. Thus, carrier trapping into band gap states appears to be responsible for the delayed current onset. This behavior will be discussed later on.

\section{DISCUSSION}

In order to study the experimentally found transient characteristics including the long delay times, the switching-on behavior of the $n-i-p-i-i-n$ device was simulated numerically. Besides the photocurrent response of the device the spatial distribution of the electron and hole current as well as the free carriers was investigated. In order to explain characteristic features in the transient behavior of the detector, it is useful to regard it as two separated, antiserially connected diodes, as already mentioned above.

Figure 6 shows the simulated photocurrent response after switching on blue light at various bias voltages. The experimentally found tendencies and characteristic features including the long delay times are well reproduced. In the following, the two cases of -1.5 and $+0.6 \mathrm{~V}$ are discussed in more detail. Thereby, two aspects of the current onset are discussed separately: first the current-voltage $(I-V)$ behavior of the top and bottom diodes, demonstrated by the blue response at a bias of $-1.5 \mathrm{~V}$, where a sign reversal of the photocurrent is observed (Fig. 4), and second at $+0.6 \mathrm{~V}$. In the latter case a delayed but monotonous rise of the photocurrent is detected. In addition, the red response at a bias voltage of $-1.5 \mathrm{~V}$ is briefly discussed later on.

\section{A. Blue response at $-1.5 \mathrm{~V}$}

In the case of blue light exposure at a bias voltage of $-1.5 \mathrm{~V}$ the photocurrent response exhibits a change from positive to negative values, with a delay of several milliseconds before steady state is reached. In order to explain this,

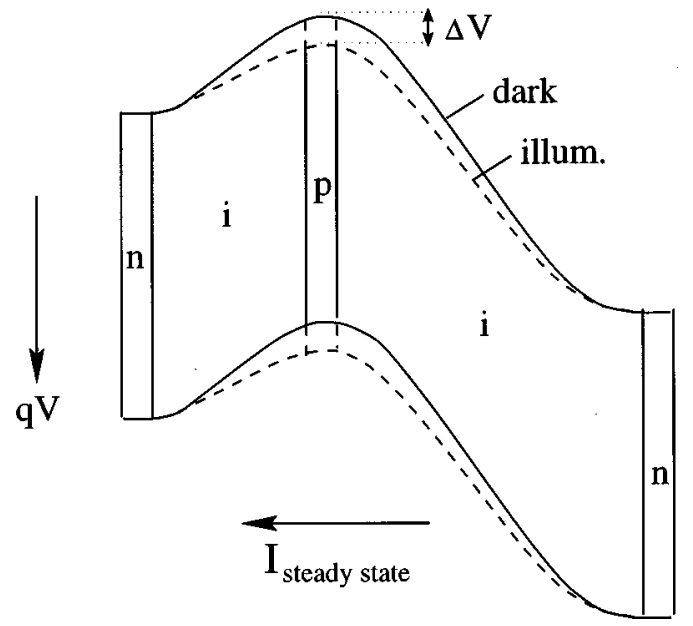

FIG. 7. Simplified band scheme in the dark (solid line) and under illumination (dashed line) with a bias voltage of $-1.5 \mathrm{~V}$.

the $I-V$ behavior of the top and bottom diode after light exposure is regarded. The voltage drops across the individual diodes $\left(V_{\text {top }}, V_{\text {bottom }}\right)$ are determined by the deviation of the electrical potential in the three doped layers from the equilibrium state. These potential drops depend on the difference between the potential at the front contact ( $n$ layer) and the central $p$ layer $\left(V_{\text {top }}\right)$, on the one hand, and between the $p$ layer and the rear contact $\left(V_{\text {bottom }}\right)$ on the other. Furthermore, the space charge distribution within the diodes plays an important role. This space charge is caused by the fixed dopands in the $p$ and the $n$ layers as well as by charged defect states in the band gap. Their charge state depends on the energetical position of the quasi-Fermi levels. Therefore, the charge determines the so-called "built-in potential" in the two diodes and, moreover, the potential in the $p$ layer. While the potential difference between the front and the rear contact is given by the externally applied voltage $V_{\text {bias }}$ and thus is constant in time, simulations reveal that the potential in the $p$ layer shifts during current onset by $\Delta V=0.15 \mathrm{~V}$. The bias voltage $V_{\text {bias }}=V_{\text {top }}+V_{\text {bottom }}$ is constant, while the individual voltages $V_{\text {top }}$ and $V_{\text {bottom }}$ are time dependent. Figure 7 shows this voltage shift schematically in a simplified band gap diagram (neglecting the specific band gap profile of the $n-i-p-i-i-n$ detector). In the central part of the detector the height of the potential barrier for electrons is reduced due to recharging of defect states. The influence of the space charge effects on the carrier transport will be explained later on. The potential change is found to take place in the time interval between 1 and $10 \mathrm{~ms}$ using a photon flux of $10^{15} \mathrm{~cm}^{-2} \mathrm{~s}^{-1}$. It coincides with the sign reversal in the photocurrent onset as shown in Figs. 4 and 7.

In order to demonstrate the consequence of this potential shift, Figs. 8 and 9 schematically show the $I-V$ behavior of the top and bottom diodes, namely $-I\left(-V_{\text {top }}\right)$ and $I\left(V_{\text {bottom }}\right)$. In the case of the top diode (Fig. 8) the negative values of current and voltage are regarded. This is done to make a direct comparison of the characteristics of both antiserially connected diodes easier. The three marking dots correspond to the dots in the experimental results (Fig. 4) and describe the transient behavior of the operation points of the 


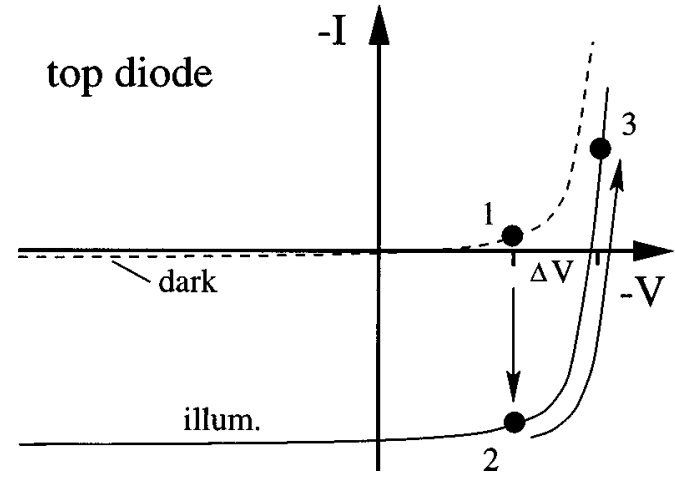

FIG. 8. Schematical $I-V$ behavior of the top diode after switching-on of illumination $(\lambda=450 \mathrm{~nm})$; in the dark (1), after $10^{-4} \mathrm{~s}(2)$, in steady state (3).

individual diodes. Since the applied bias voltage at the detector is kept constant $(-1.5 \mathrm{~V})$ the sum of the voltages at the top and bottom diode must also be constant. Dot 1 in Figs. 8 and 9 indicates the $I-V$ values of the diodes in the dark $(t=0)$. It correlates with the experimental results shown in Fig. 4. Dot 2 marks the delayed photocurrent rise $\left(t=10^{-4} \mathrm{~s}\right)$. Dot 3 denotes the steady state for $t>10^{-2} \mathrm{~s}$.

First the behavior of the bottom diode is discussed in more detail (Fig. 9). Before switching on the light, the current in the reverse biased bottom diode is a thermally activated generation current (dot 1). Under light exposure additional carriers are generated resulting in a photogenerated current (dot 2). In the time interval between $10^{-4} \mathrm{~s}$ and steady state ( $\operatorname{dot} 2 \rightarrow \operatorname{dot} 3)$ the potential in the $p$ layer is reduced by $\Delta V$. In steady state the $I-V$ value of the bottom diode reaches dot 3 . However, the photocurrent of the bottom diode is not affected significantly, because the diode remains reverse biased and, thus, all photogenerated carriers are collected.

In contrast to this, the top diode is forward biased (Fig. 8). This means that the dark current is a diffusion current before light exposure (dot 1). Electrons and holes are injected from the front $n$ layer and the central $p$ layer into the top diode, respectively. Thus, the total current is limited by holes, which are generated in the bottom diode and injected through the $p$ layer into the top diode. The dark current in the

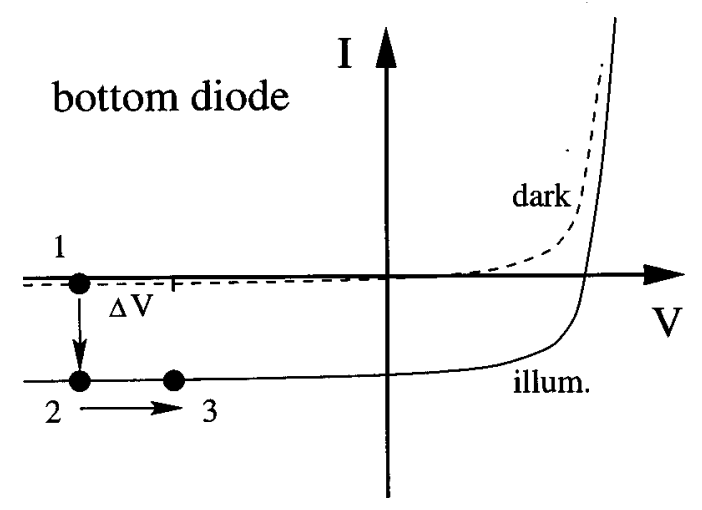

FIG. 9. Schematical $I-V$ behavior of the bottom diode after switching on of illumination $(\lambda=450 \mathrm{~nm})$ : in the dark (1) after $10^{-4} \mathrm{~s}(2)$, in steady state (3).

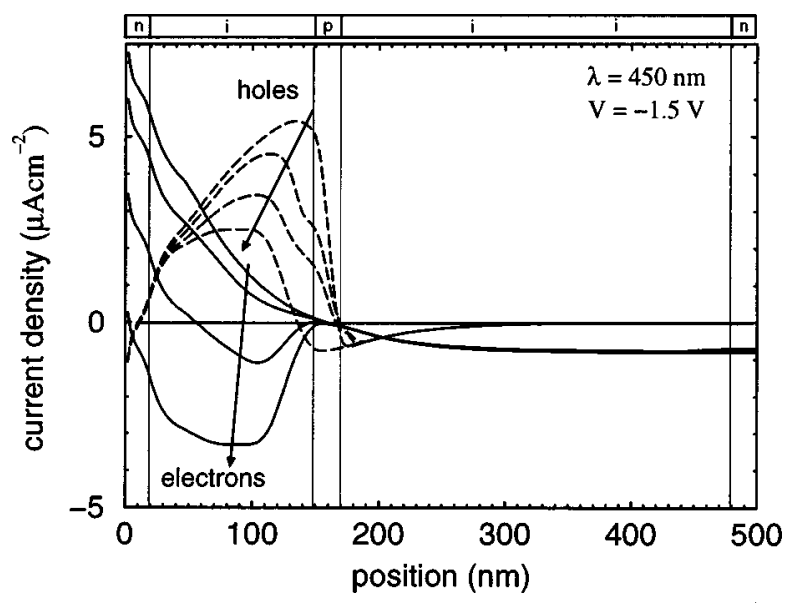

FIG. 10. Electron and hole current densities after $10^{-4}, 10^{-3}, 2 \times 10^{-3}$, and $10^{-2}$ with a bias voltage of $-1.5 \mathrm{~V}$.

bottom diode determines the dark current in the top diode. In the time regime after light exposure and before the voltage shift is observed, the $I-V$ value of the top diode lies in the active quadrant (dot 2). As in a photodiode, the photogenerated carriers are collected, which results in a positive photocurrent. Due to the spatially inhomogeneous generation profile, caused by the strong absorption of blue light, this positive photocurrent in the top diode reaches a high value and is, thus, overcompensating the negative photocurrent in the bottom diode. Consequently, in this time interval a positive total current is detected (Fig. 4). Later on, the operation point of the top diode moves from dot 2 to dot 3 and the top diode does not generate a photocurrent, due to the voltage shift by $\Delta V$. Consequently, the total current changes from positive to negative values (Fig. 4). In steady state the current is again determined by the low rate of photogenerated carriers in the bottom diode (dot 3$)$.

More insight into this transient behavior is attained from the spatial distribution of the electron and hole current regarded as $10^{-4}, 10^{-3}, 2 \times 10^{-3}$, and $10^{-2} \mathrm{~s}$ after switching on illumination, which results from simulations (Fig. 10). In the case of a positive hole current, holes move in the $x$ direction, while a positive electron current means that electrons move in reverse direction and vice versa. Figure 10 shows that for early times in both diodes electrons move toward the two $n$ layers and holes toward the central $p$ layer. Thus, carrier collection by the electric field occurs in both diodes and the current is drift controlled. Since under blue light illumination most carriers are generated in the top diode, the positive current in the top diode overcompensates the negative current in the bottom diode and, therefore, the total current is positive. For later times both diodes behave differently. While in the bottom diode the current distribution remains unchanged, namely negative and drift controlled, in the top diode it varies remarkably. Due to the potential shift in the central $p$ layer more and more electrons diffuse from the front contact back toward the $p$ layer. Thus, the sign of the electron current changes from positive to negative. In contrast, the hole current is affected less drastically and changes its sign only in the vicinity of the $p$ layer. However, 
before steady state is reached the hole current in the $p$ layer is positive. This indicates that photogenerated holes move from the front and middle part of the top diode $i$ layer toward the $p$ layer because blue light is preferentially absorbed there. The few holes which are collected in the bottom diode drift toward the $p / i$ interface region of this diode. The transient transport behavior of holes describes the collection of positive charge (holes) in the $p$ layer and can be attributed to a refilling of neutralized defect states $\left(D^{p}\right)$ there. This causes a potential shift in the $p$ layer and leads to a reduction of the electric field and, therefore, to a reduced drift of holes in the $p / i$ interface region of the top diode. Finally, in steady state, the current in the top diode is a recombination current with a maximal recombination rate in the vicinity of the $p$ layer at a position of $120 \mathrm{~nm}$. Electrons which are injected at the front contact, therefore, recombine with holes which are photogenerated and collected in the bottom diode and injected through the $p$ layer. The majority of carriers which are generated in the bottom diode are collected by the electric field.

The investigations have shown that the high number of defect states (dangling bonds) significantly influences the transient response of the $n-i-p-i-n$ structure in the region of the central $p$ layer. Under this condition the $I-V$ characteristics of the $n-i-p-i-n$ structure behave like two antiserially connected diodes. A decrease of the conductivity in the central $p$ layer due to reduced doping concentration or a decrease of the $p$ layer thickness leads to a reduction of $D^{p}$ states (Fig. 2) as a consequence of the Fermi level dependent defect state distribution. ${ }^{19}$ However, in this case a more ohmic $I-V$ characteristic of the $n-i-p-i-n$ structure is observed, ${ }^{12}$ since for higher bias the $p$ layer is flooded with electrons ${ }^{12}$ and the dynamic range as well as the color separation decrease significantly.

\section{B. Blue response at $+0.6 \mathrm{~V}$}

In the following the second aspect of the photocurrent onset, namely the delayed but monotonous rise of the photocurrent, is investigated. Therefore, we applied a bias voltage of $+0.6 \mathrm{~V}$. In this case the top diode is reverse biased and the bottom diode is forward biased and a monotonous photocurrent rise after light exposure is observed (Fig. 6). Figure 11 shows the simulated electron and hole current densities. As in the case of $V_{\text {bias }}=-1.5 \mathrm{~V}$ the current is drift controlled in both diodes for early times $\left(t<10^{-4} \mathrm{~s}\right)$. Afterwards, in the time interval from $10^{-4}$ to $10^{-2} \mathrm{~s}$, the bottom diode switches from blocking to permeable, which means that more and more holes are injected from the top diode through the $p$ layer into the bottom diode and electrons from the back contact into the bottom diode, and the current becomes dominated by diffusion. In steady state the current in the bottom diode is nearly a pure recombination current. Electrons and holes recombine via the enhanced defect density in the rear part of the bottom diode. The main recombination takes place in the low band gap region between 370 and $460 \mathrm{~nm}$ where the defect density is significantly higher than in the front part (Fig. 2). As in the case of $V_{\text {bias }}=-1.5 \mathrm{~V}$ the delayed stepwise current change can be explained by a switching of the forward biased diode, which is in this case the

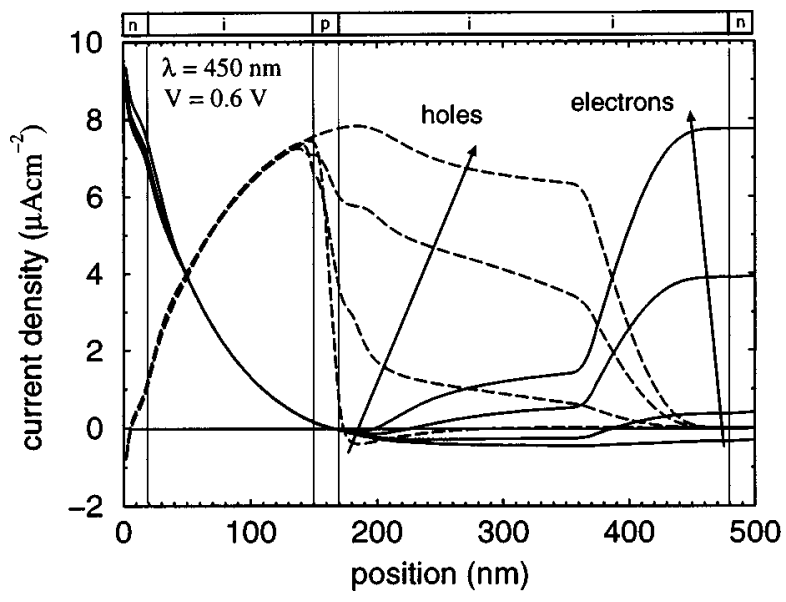

FIG. 11. Electron and hole current densities after $10^{-4}, 10^{-3}, 2 \times 10^{-3}$, and $10^{-2} \mathrm{~s}$ with a bias voltage of $+0.6 \mathrm{~V}$.

bottom diode. As already mentioned this coincides with a remarkable potential shift in the central $p$ layer. However, the question remains by which mechanism this potential shift, and thus the switching behavior, is controlled.

Further insight into this mechanism can be obtained from the spatial distribution of free electrons and holes, shown in Fig. 12. In the dark as well as under light exposure the electron density is high $\left(10^{15} \mathrm{~cm}^{-3}\right)$ in the $n$ layers, where electrons are majority carriers. It is low $\left(10^{6} \mathrm{~cm}^{-3}\right)$ in the $p$ layer where they are the minorities. Moreover, one finds no significant time dependent variation of the electron concentration within the device. The high gradients in the electron concentration in the rear part of the device result from the band gap grading. Since only conduction-band edge shifting was assumed in the simulations, the density of free electrons is distinctly affected by the grading. For the hole concentration a different behavior is found. Although holes are the majorities in the $p$ layer, their density is very low in the dark $\left(10^{8} \mathrm{~cm}^{-3}\right)$. The reason for this is a depletion of the $p$ layer after applying a bias voltage. In this case not only the hole concentration is drastically reduced, but also the positively charged defect states in the $p$ layer are partly neutral-

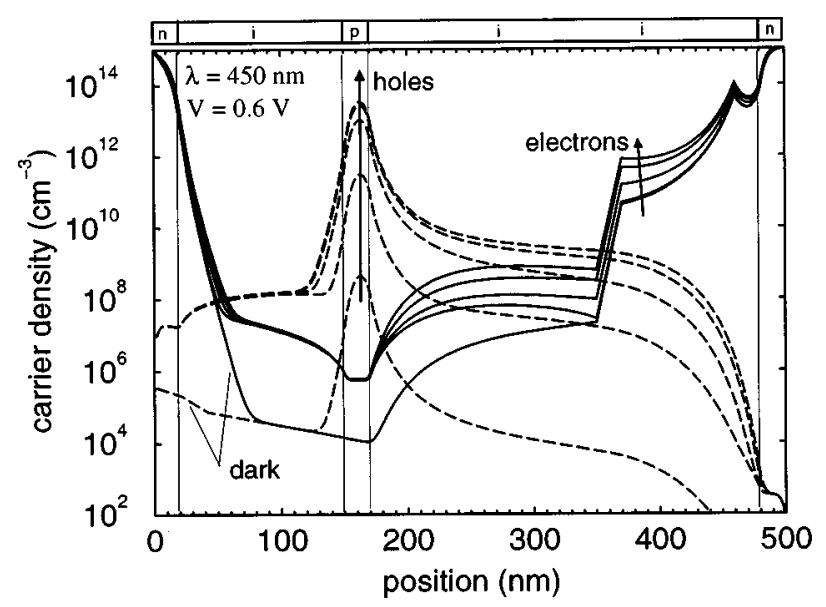

FIG. 12. Electron and hole densities in the dark and after $10^{-4}, 10^{-3}, 2$ $\times 10^{-3}$, and $10^{-2} \mathrm{~s}$ with a bias voltage of $+0.6 \mathrm{~V}$. 
ized compared with equilibrium (Fig. $2 D^{p}$ peak). Thus, the positive charge which partly compensates the space charge of the negatively charged acceptor states, originated from doping, is reduced. This results in a temporary enhanced built-in potential in the top and bottom diodes.

Under illumination the $p$ layer is gradually refilled with photogenerated holes drifting predominantly from the top diode toward the $p$ layer. After about $10^{-2} \mathrm{~s}$ this recharging process is completed and the hole density reaches a high value of $10^{13} \mathrm{~cm}^{-3}$. As a consequence of this increase in the hole density over several orders of magnitude, the quasiFermi level of holes moves toward the valence-band edge. This results in a significant recharging of defect states in and near the $p$ layer from neutral to positive by hole trapping, because these defect states are located in the lower half of the band gap. The increase of positive space charge finally explains the above discussed shift of the electrical potential in the $p$ layer by $\Delta V$ (Fig. 7). Thus, the explanation for the delayed current onset can be given as follows: holes are trapped into defect states in and near the $p$ layer, recharging them from neutral to positive. Afterwards they diffuse through the $p$ layer into the forward biased diode. The recharging process reduces the potential barrier for electrons in the $p$ layer; more electrons diffuse from the contact toward the $p$ layer and, thus, becomes available for recombination with holes. The simulations reveal that this behavior, including the sign reversal after light exposure for certain bias voltages, is a device structure inherent phenomenon originating from the depleted $p$ layer in the dark.

From another point of view the recharging process in the reverse biased top diode during current onset is equivalent to the recharging of a capacitor. While the electron and hole current is nearly time independent in this diode (Fig. 10), the recharging of defect states in and near the $p$ layer results in a potential shift and, therefore, in a high displacement current. This displacement current is typical for the charging of a capacitor. The charging process is expected to be faster with a higher optical generation rate, because more holes are generated and the recharging rate is higher. Indeed, a scaling of the current-onset time with the generation rate could be shown previously. ${ }^{15}$ The transient behavior of the color detector is limited by the refilling rate of defect states. To achieve shorter delay times in the transient behavior of the detector this capacitance has to be reduced. Therefore, the influence of the doping concentration and the thickness of the individual layers on the transient response will be addressed in the future to speed up the detector.

\section{Red response}

Figure 13 shows the simulated transient response after light exposure with a wavelength of $650 \mathrm{~nm}$. Under these conditions the dominant light absorption takes place in the rear low-band gap part of the device. The experimentally found characteristics (Fig. 5) are well reproduced. In spite of a more complex transient photocurrent behavior the response exhibits a delay of the current onset by several milliseconds, which was also found in the case of illumination with blue light. Simulations of the electron and hole current show be-

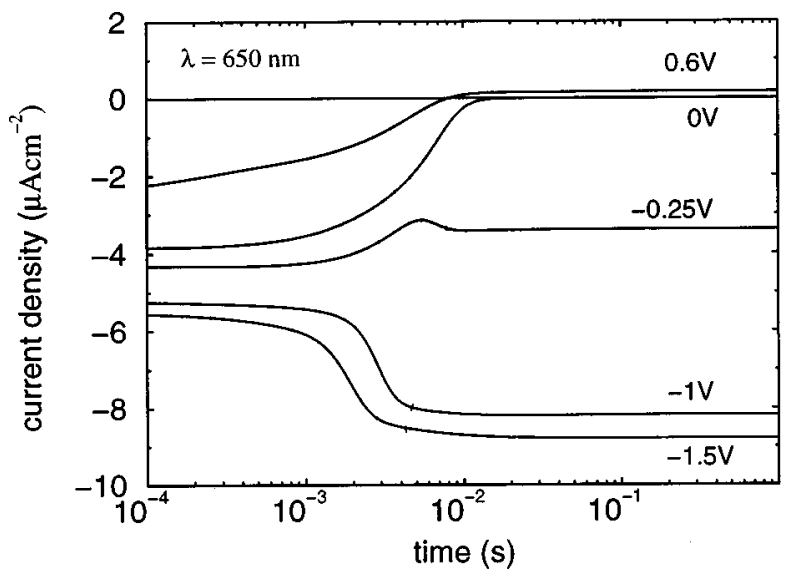

FIG. 13. Simulated transient photocurrent response to switching on of red illumination $(\lambda=650 \mathrm{~nm})$.

havior corresponding to that under blue light exposure. For $V_{\text {bias }}=-1.5 \mathrm{~V}$ most carriers are generated and collected in the reverse biased bottom diode. The current in this diode leads to the recharging of defect states in and near the central $p$ layer with holes. Therefore, the potential is shifted in the $p$ layer and the forward biased top diode switches from blocking to permeable. This finally results in the same process as described for the case of blue light.

\section{CONCLUSIONS}

The transient behavior of three-color detectors was investigated by experimental and theoretical studies of the transient photocurrent response after switching on monochromatic illumination. Good agreement between experimental results and numerical simulations could be achieved. Phenomena like a delayed current onset and a sign reversal in the transient photocurrent response are explained by defect recharging by trapped holes in the vicinity of the central $p$ layer. As a result, a remarkable potential shift in the $p$ layer is observed in the simulation causing a switching of the forward biased diode from blocking to permeable. The sign reversal of the photocurrent after light exposure is attributed to a compensation or overcompensation of the photocurrent of the reverse biased diode by the presumably forward biased diodes, due to the depletion of the $p$ layer in the dark. However, the strong illumination dependence of the transient response after light switching complicates the application of $n-i-p-i-n$ structures for color detectors.

\section{ACKNOWLEDGMENTS}

The authors thank R. Carius, J. Fölsch, and J. Zimmer for helpful discussions. Funding by the Bundesministerium für Bildung, Forschung und Technologie (BMBF) is gratefully acknowledged.

${ }^{1}$ H. K. Tsai and S. C. Lee, Appl. Phys. Lett. 52, 275 (1988).

${ }^{2}$ H. Stiebig, J. Giehl, D. Knipp, P. Rieve, and M. Böhm, Mater. Res. Soc. Symp. Proc. 377, 815 (1995).

${ }^{3}$ D. Caputo, F. Irrera, F. Palma, S. R. Rachele, and M. Tucci, J. Non-Cryst. Solids 198-200, 1172 (1996).

${ }^{4}$ D. Knipp, H. Stiebig, J. Fölsch, R. Carius, and H. Wagner, Mater. Res. Soc. Symp. Proc. 467, 735 (1997). 
${ }^{5}$ T. Neidlinger, M. B. Schubert, G. Schmid, and H. Brummack, Mater. Res. Soc. Symp. Proc. 420, 147 (1996).

${ }^{6}$ D. Caputo, F. Irrera, F. Palma, S. R. Rachele, and M. Tucci, J. Non-Cryst. Solids 198-200, 1172 (1996).

${ }^{7}$ M. Topic, F. Smole, J. Furlan, and W. Kusian, J. Non-Cryst. Solids 198200, 1180 (1996).

${ }^{8}$ R. A. Street, X. D. Wu, R. Weisfield, S. Ready, R. Apte, D. K. Biegelsen, W. B. Jackson, and P. Nylen, J. Non-Cryst. Solids 198-200, 1151 (1996).

${ }^{9}$ M. Okamura, K. Kimura, S. Shirai, and N. Yamauchi, IEEE Trans. Electron Devices 41, 180 (1994).

${ }^{10}$ S. Manabe et al., IEEE Trans. Electron Devices 38, 1765 (1991).

${ }^{11}$ Q. Zhu, H. Stiebig, P. Rieve, H. Fischer, and M. Böhm, Mater. Res. Soc. Symp. Proc. 336, 843 (1994).

${ }^{12}$ H. Stiebig and M. Böhm, J. Non-Cryst. Solids 164-166, 785 (1993).

${ }^{13}$ H. Wieczorek, J. Non-Cryst. Solids 164-166, 781 (1993).

${ }^{14}$ D. Knipp, H. Stiebig, J. Fölsch, F. Finger, and H. Wagner, J. Appl. Phys. 83, 1463 (1998)
${ }^{15}$ H. Stiebig, C. Ulrichs, T. Kulessa, J. Fölsch, F. Finger, and H. Wagner, J. Non-Cryst. Solids 198-200, 1185 (1996).

${ }^{16}$ H. Stiebig, D. Knipp, J. Zimmer, and H. Wagner, IEEE Trans. Electron Devices 47, 1438 (1998).

${ }^{17}$ W. Luft and Y. S. Tsuo, Hydrogenated Amorphous Silicon Alloy Deposition Processes (Marcel Dekker Inc., New York, 1993).

${ }^{18}$ J. Fölsch, F. Finger, T. Kulessa, F. Seibke, W. Beyer, and H. Wagner, Mater. Res. Soc. Symp. Proc. 377, 517 (1995).

${ }^{19}$ H. Stiebig and F. Siebke, Philos. Mag. B 72, 489 (1995).

${ }^{20}$ R. Carius, H. Stiebig, F. Siebke, and J. Fölsch, J. Non-Cryst. Solids (in press).

${ }^{21}$ H. Stiebig, Th. Eickhoff, J. Zimmer, C. Beneking, and H. Wagner, Mater. Res. Soc. Symp. Proc. 420, 855 (1996).

${ }^{22}$ J. Zimmer, H. Stiebig, and H. Wagner, J. Appl. Phys. 84, 611 (1998).

${ }^{23}$ B. Stannowski, H. Cordes, R. Brüggemann, Th. Eickhoff, S. Bröcheler, and H. Wagner, J. Non-Cryst. Solids 227-230, 1295 (1998). 